\title{
Extrapericardial tamponade due to gastric dilatation: roll of bedside focused cardiac ultrasound
}

\author{
Marta Caballero Milán, Marc Vives Santacana. \\ Department of Anesthesiology, Resuscitation and Pain Therapy; Bellvitge University Hospital; Hospitalet de Llobregat; Barcelona.
}

\section{Introduction}

Extrapericardial tamponade is a life-threatening compression of the heart with an extrapericardial etiology. Common causes of extrapericardial tamponade are hematoma, tumors, ascites, and hernias. This report describes the case of a 75-year-old man with an obstructive shock secondary to a very large gastric dilatation compressing the left ventricle, who was diagnosed by a Focused Cardiac Ultrasound (FCU) which led to gastric decompression improving hemodynamics and reversing the circulatory shock state.

\section{Case}

A 75-year old man underwent cystoprostatectomy, lymphadenectomy and bilateral nephroureterectomy. He had hypertension, diabetes, dyslipidemia, hypertensive cardiomyopathy, hyperuricemia, chronic kidney disease on dialysis, intermittent claudication with right femoropopliteal bypass and left supracondylar amputation.

48 hours after surgery, the patient started to become progressively hemodynamically unstable: hypotension $(70 / 30 \mathrm{~mm} \mathrm{Hg})$, tachycardia (>110 bpm), a central venous pressure (CVP) of $14 \mathrm{mmHg}$, dyspnea, abdominal pain, pale skin, feeling of impending doom and an $\mathrm{Hb}$ of 8.1 (hematocrit of 24\%).

- Bedside Focus Cardiac Ultrasound (FCU): systolic and diastolic obliteration of the Left Ventricle (LV) [Figure 1] with preserved contractlity was observed. An intra-abdominal hypoechoic image, oriented as free liquid compressing the LV, was also observed. Hypotension was refractory to transfusion of two packed red blood cells and infusion of noradrenaline at $0.1 \mathrm{mcg} / \mathrm{kg} / \mathrm{min}$.

- Thoracoabdominal CT scan: important gastric dilatation and first to second duodenal portion with plenty of liquid inside [Figure 2-3] were observed in the CT scan, which confirmed our suspicion.

A nasogastric tube was inserted and $1,700 \mathrm{cc}$ of bilious fluid were drained [Figure 4]. Subsequently, patient hemodynamics significantly improved weaning off vasopressors, and an improvement in the signs of tissue perfusion were observed.

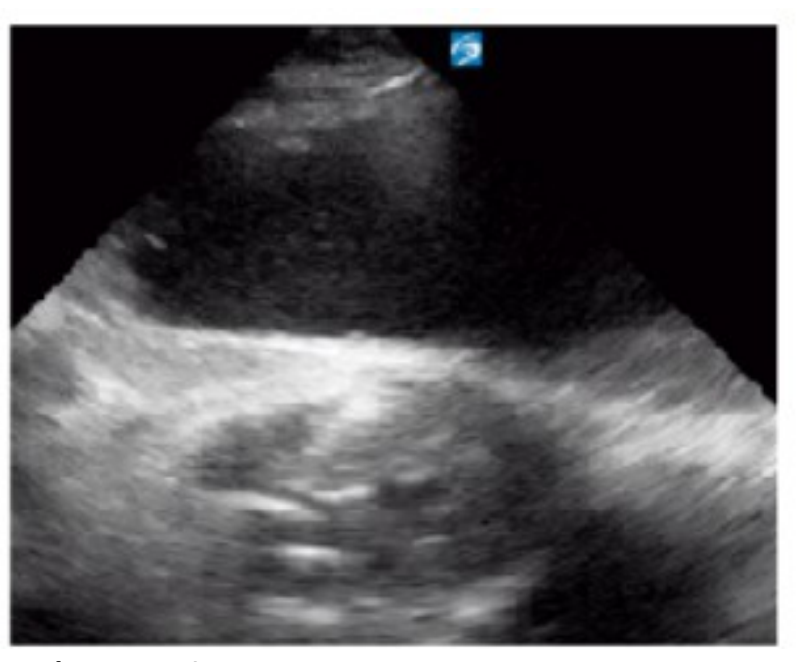

Figure 1

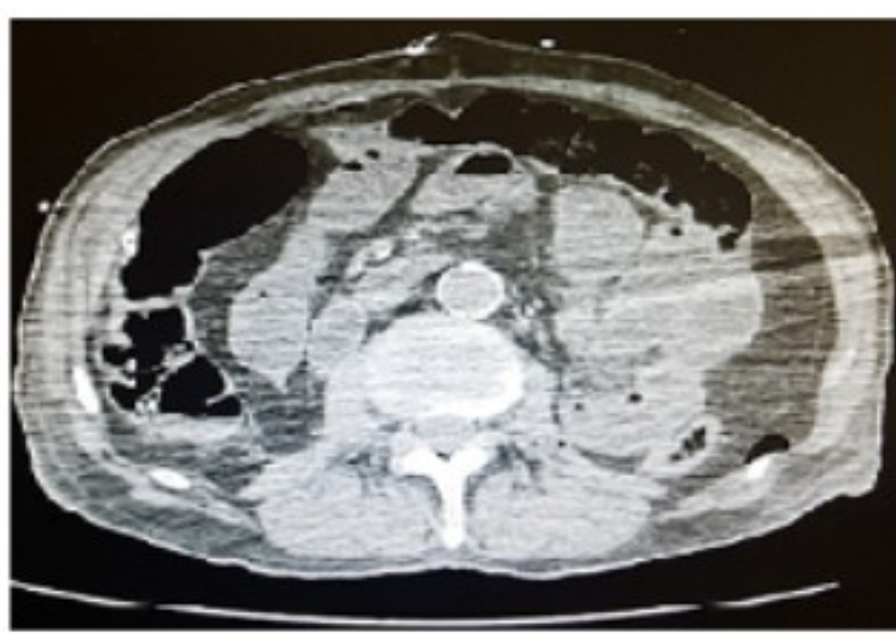

Figure 3

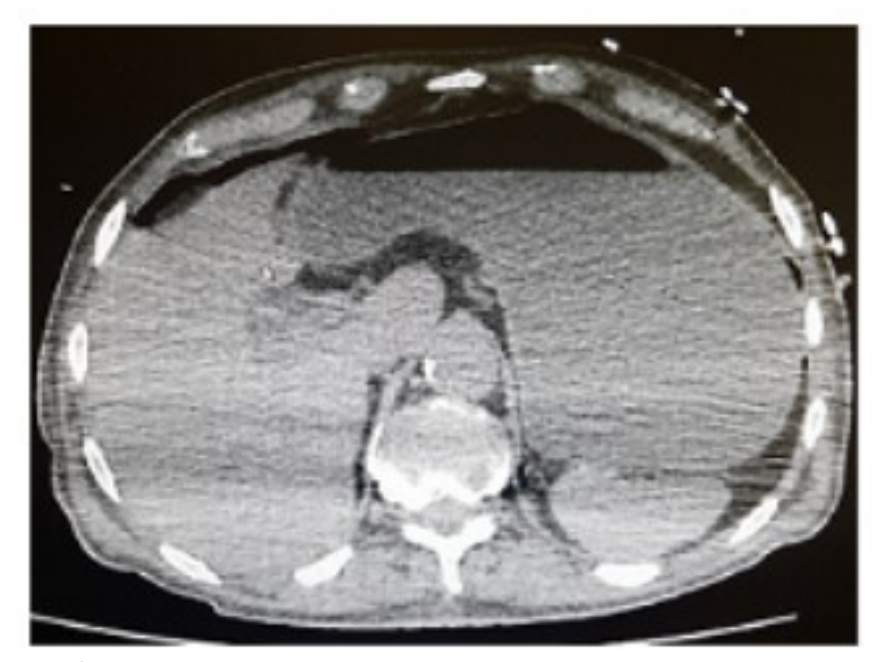

Figure 2

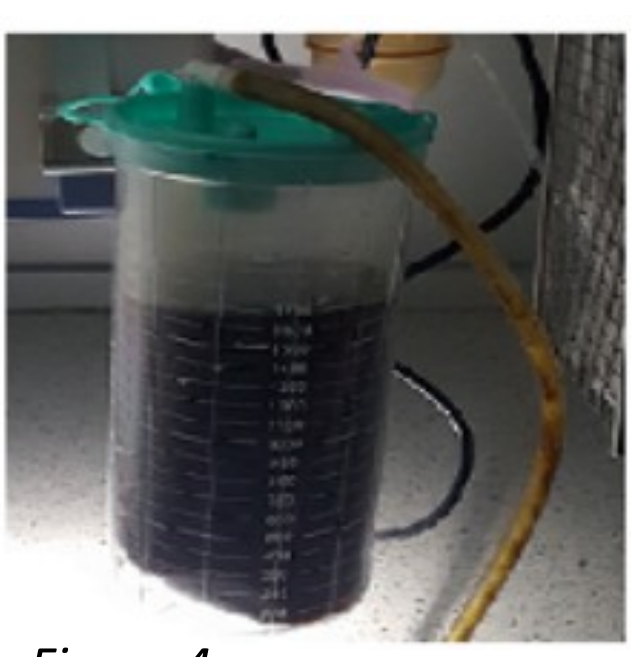

Figure 4

\section{Discussion}

Cardiac tamponade usually occurs due to intrapericardial causes, such as idiopathic pericarditis, uremia, and bleeding [1]. More rarely, cardiac tamponade develops when hematoma, tumors, or ascites, which may originate from the extrapericardial area, compress the heart and great vessels [2].

Diagnosis of cardiac tamponade is based on clinical signs. However, bedside FCU may definitely help in the diferential diagnosis of the cause of hypotension and therefore leading to early treatment. It may be difficult to diagnose cardiac tamponade when the diagnostic results are unclear, as in cases with extrapericardial causes.

In patients with hemodynamic instability, FCU in these setting clearly adds to the bedside physical examination and can be performed immediately at the bedside [3].

FCU has been shown to be helpful to traditional bedside assessment in determining volume status and LV systolic function.

In patients who are hemodynamically unstable, FCU diagnoses may impact therapy in terms of the use of volume repletion, vasopressors, and inotropes, as well as diuretics and vasodilators.

Findings on an FCU examination should not be considered definitive, but integrated with the other bedside patient data (history and physical examination) to form an initial diagnostic impression that can then be pursued with alternative imaging or diagnostic modalities.

Management of extrapericardial tamponade varies depending on the underlying condition, but definitive treatment usually requires the removal of compressive materials. The moderately increase in CVP associated with hypotension and tachycardia triggered the suspicion of cardiac tamponade in our case.

Previous cases of cardiac tamponade due to extrapericardial causes have been reported. However, is relatively uncommon in critically ill patients.

\section{Conclusion}

Bedside FCU is a very useful tool for an early diagnosis leading to an appropiate timely manner treatment.

Although extrapericardial LV compression secondary to gastric dilatation is not a common cause, it should be taken into consideration in the differential diagnosis, especially in the postoperative period with the associated higher risk of paralytic ileus.

[1]Spodick DH. Acute cardiac tamponade. N Engl J Med 2003;349:684-90. [2]Coleman GM, Fischer $R$, Fuentes F. Blunt chest trauma. Extrapericardial cardiac tamponade by a mediastinal hematoma. Chest 1989;95:922-4.

[3]Labovitz AJ, Noble VE, Bierig M, Goldstein SA, Jones R, Kort S et al. Focused cardiac ultrasound in the emergent setting: a consensus statement of the American Society of Echocardiography and American College of Emergency Physicians. I Am Soc Echocardiogr: official publication of the American Society of Echocardiography 2010;23:122530.

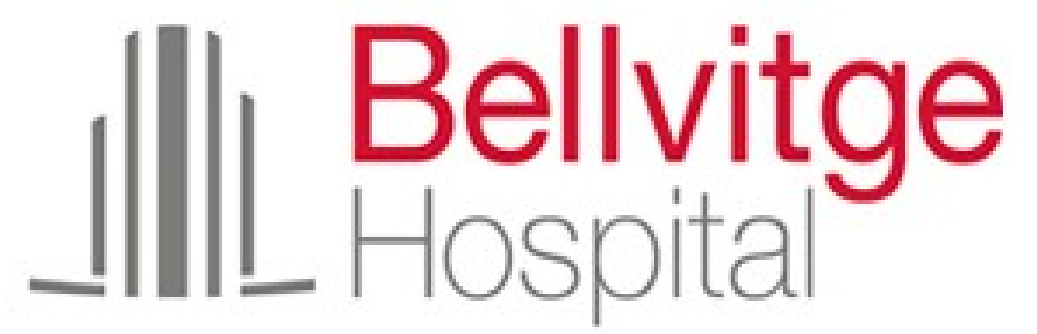

